\title{
Hepatic tumor enhancement in computed tomography: combined models of liver perfusion and dynamic imaging
}

\author{
Johanne Bézy-Wendlinga,*, Marek Kretowski ${ }^{\mathrm{a}, \mathrm{b}}$, Yan Rolland ${ }^{\mathrm{a}, \mathrm{c}}$ \\ ${ }^{a}$ Laboratoire Traitement du Signal et de l'Image, INSERM, Bâtiment 22, Campus de Beaulieu, Université de Rennes \\ 1, 35042 Rennes Cedex, France \\ ${ }^{\mathrm{b}}$ Institute of Computer Science, Technical University of Bialystok, Wiejska 45a, 15-351 Bialystok, Poland

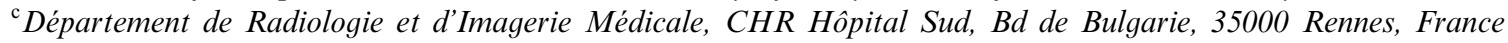

Received 4 March 2002; accepted 11 June 2002

\begin{abstract}
The objective of this study is to show how computational modeling can be used to increase our understanding of liver enhancement in dynamic computer tomography. It relies on two models: (1) a vascular model, based on physiological rules, is used to generate the 3D hepatic vascular network; (2) the physical process of CT acquisition allows to synthesize timed-stamped series of images, aimed at tracking the propagation of a contrast material through the vessel network and the parenchyma. The coupled models are used to simulate the enhancement of a hyper-vascular tumor at different acquisition times, showing a maximum conspicuity during the arterial phase.
\end{abstract}

(C) 2002 Elsevier Science Ltd. All rights reserved.

Keywords: Dynamic CT; Computational modeling; Tumor conspicuity; Liver enhancement

\section{Introduction}

Hepatic hemodynamic interactions are very complex, especially because of the particular perfusion of the liver. This organ is irrigated by a dual blood supply: $20-25 \%$ of the blood comes directly from the aorta, through the hepatic artery (HA) and 75-80\% from the mesentery through the portal vein (PV). At the entry of the hepatic capillaries (sinusoids) the two flows mix. Dual-phase helical CT (arterial and portal phases) is commonly used to detect vascular lesions, and especially hyper- and hypo-vascular tumors. The contrast material (CM) generally injected in a vein of the arm reaches the liver with a delay between the HA and the PV. This explains that the local enhancement of the

* Corresponding author.: Tel.: +33-2-23-23-62-20; fax: +33-2-23-23-69-17.
E-mail address: johanne.bezy@univ-rennes1.fr (J. Bézy-Wendling). 
liver is very dependent on the region perfusion: regions irrigated only or mainly by the HA will enhance differently than regions perfused by both of the supplying trees, leading to a widening of the difference in attenuation between normal parenchyma and tumors.

However, the tumor conspicuity is strongly dependent on intrinsic (anatomical, patho-physiological) and extrinsic (injection, acquisition) parameters. The control of these parameters is needed to correctly study their influence on the visual appearance of tumors in images, but also on the automatic characterization of abnormal processes by image analysis methods. This control is difficult to obtain in vivo, especially for intrinsic parameters.

A computational model of hepatic enhancement in CT is proposed in this paper. It departs from previous contributions [1,2] and allows to analyze the propagation of contrast medium in the three hepatic vascular trees (HA, PV and Hepatic Vein-HV). It combines (i) the modeling of the liver (parenchyma and vascular network) allowing to change anatomical and physiological parameters and (ii) the modeling of $\mathrm{CT}$ acquisition, where the main acquisition parameters as well as injection modalities can be changed. The influence of all these parameters on the image characteristics can then be studied.

The remaining parts of this paper are organized as follows. Section 2 briefly reports a state of the art in liver enhancement understanding. Section 3 provides a description of the proposed models of perfusion and CT imaging. Experimental results in normal and pathological situations are displayed in Section 4. Discussions and some plans for future research are sketched in the last section.

\section{State of the art}

Spiral CT scanning after injection of contrast medium provides high quality images of the liver and the capability to fully explore the organ during arterial and portal phases. It improves the detection and the characterization of hepatic tumors, by amplifying the difference in attenuation between the tumor and the normal parenchyma [3,4]. Distinction of the two main phases of the liver enhancement is of great clinical interest: it allows to determine the optimal temporal windows for the liver exploration, and for a maximal conspicuity of tumors. For example, hyper-vascular hepatic lesions are better detectable during the pure arterial phase when the contribution of the portal blood supply is not preponderant yet. But the duration of this phase is difficult to estimate [5]. This is mainly due to the variations of the hepatic enhancement, with a large number of parameters (type and quantity of contrast material, injection flow and duration, monophasic or biphasic injection) or the tumor types [6]. Many clinical studies have been performed during the last 10 years, in order to understand the influence of all these features. For instance, in [7], it is shown on a group of 109 patients that the risk of missing a tumor decreases with a double phase acquisition, compared to a simple one. In [8], the influence of the quantity of contrast product, and the injection flow is evaluated on 25 patients, concluding that augmenting the $\mathrm{CM}$ flow results in an acceleration of the hepatic enhancement.

Dodd and Baron [9] underline the lack of standardization of clinical studies, whose results are difficult to compare. Indeed, in some of these studies, extrinsic parameters (depending of the CM administration and the image acquisition protocol, number of slices, level of slices...) are often heuristically chosen with an important part of randomness, and significant intrinsic parameters can be ignored. The necessity to control these parameters is still amplified by the fast advances that intervene 
in the imaging technology as well as in the field of contrast materials, limiting the usefulness of clinical studies. Few models of the liver enhancement have been proposed so far. Bae et al. [10] applied their compartmental model of the human cardiovascular system to simulate enhancement curves corresponding to three groups of patients, with varying size and weight. The computer model presented in [11] is also interesting to quantify the hepatic perfusion, and to extract anatomical and functional properties from a CT acquisition. Work of Kim et al. [12] is based on a compartmental model of the liver, including a tumor. This model is used to simulate enhancement curves, and to predict the optimal injection protocol. All these models are useful to simulate curves corresponding to the whole liver but cannot be used to synthesize images, or local vascular modifications.

Modeling of the vascularization is the first step required to create realistic CT scans. 3D functional models have been presented in [1,13]. The model [1] was extended to allow infusion of CM and its propagation from the main supplying vessels (hepatic artery, portal vein) to the output hepatic vein, through the parenchyma. This model is coupled with a model of the CT modality leading to the synthesis of dynamic images of the liver, where local pathological changes can be tracked during the $\mathrm{CM}$ propagation.

\section{Modeling}

The model is used to simulate the tissue growth as well as the development of the vascular network perfusing it, in a succession of growth cycles. Then, after the generation of a vascularized liver, dynamic CT scans are simulated at different times during the propagation of the contrast product, in the vessels and the parenchyma.

\subsection{Model of the liver perfusion}

\subsubsection{Tissue}

The external shape of the simulated liver has been obtained by segmentation of a 2D CT scan acquisition (about 100 slices). The whole organ is constituted of macro-functional units, called "macro-cells" (each macro-cell is a small, fixed size part of the tissue). Several classes of macro-cells co-exist, and each of them is characterized by physiological properties (e.g. blood pressure and flow, rate of mitosis/necrosis). In the same class, small variations of certain parameters (such as the blood flow) can be used to simulate natural vascular variability due to very small vessels.

The organ size periodically increases until reaching the one of an adult liver. At each growth cycle, some macro-cells divide into two macro-cells of the same class (process of mitosis governed by a probability $\mathrm{Pb}_{\mathrm{m}}$ ), and some of them disappear (necrosis governed by parameter $\mathrm{Pb}_{\mathrm{n}}$ ). Parameters $\mathrm{Pb}_{\mathrm{m}}$ and $\mathrm{Pb}_{\mathrm{n}}$ decrease with time, in order to follow the natural process of cellular regeneration, slower at the end than at the beginning of their life.

\subsubsection{Vessels}

The modeled hepatic vascular network is made of three functionally connected trees: HA, PV and $\mathrm{HV}$. Blood circulates from HA and PV to HV, going through the macro-cells, which represent the sinusoids (hepatic capillaries), where exchanges of gases and nutrients between blood and tissue take place. Each of these three trees is a binary tree, made of a succession of branches and nodes. The 
main parameters associated to a node are its spatial position and the blood pressure at this position. A vessel is composed of consecutive segments (branch between two bifurcations) [14], which are rigid tubes with a thickness depending on the kind of vessel (artery or vein). The blood is considered as a Newtonian fluid, with a constant viscosity. Its flow is supposed to be governed by Poiseuille's law. This law is used to compute the difference of pressure between the extremities of a branch, given its geometry (length and radius) and the blood flow in it. At each bifurcation and at each time, the law of matter preservation is verified: the same blood quantity enters the bifurcation by the father vessel and leaves it by the two sons vessels. Another relation, extracted from experimental data, is used to relate the radius of the father vessel and the radii of its sons $[15,16]$.

The vascular network development is caused by the increase of blood needs, at each growth cycle. New macro-cells that are not perfused yet, signalize it by secreting some angiogenic factors (AF), which stimulate the closest vessels to sprout new vessels toward the macro-cell [17]. A local optimization process is used to compute the geometry of the new bifurcation following the criteria of "minimum volume" $[18,19]$. The next step consists in re-computing the characteristics of the whole tree (blood flow, pressure and radii). A fast algorithm has been defined to optimize this procedure, that allows to considerably reduce the computation time [20].

\subsection{Model of dynamic imaging}

Two steps are considered in the modeling of dynamic CT of the liver: (1) the propagation of the contrast material in the vascular network has to be simulated, and (2) images of a given region of the organ, can be generated at different times.

\subsubsection{Contrast medium propagation}

To create the 3D density representation of the liver, after injection of the contrast material, the concentration evolution has to be known in all vessels and macro-cells. In order to simplify the calculation, the concentration is supposed to be constant in each vessel segment and the blood is ideally mixed with the contrast material.

In clinical practice the contrast material is infused intravenously (e.g. in an antecubital vein) and after the bolus transfer time it enters the liver, first through the HA and then, with a certain delay, through the PV. Modeling separately the infusion for the HA and the PV sources, by providing the $\mathrm{CM}$ concentration curves at these two entries, gives more flexibility in the simulation of mono or bi-phasic injection with varying conditions (e.g. duration, amount of $\mathrm{CM}$ ) or features specific to a patient (e.g. delay between HA and PV).

The CM propagation in a vascular tree is based on the observation that time $\Delta t$ needed by the blood to traverse a vessel can be calculated using its length, radius and the blood flow in it (see Fig. 1a). It means that the contrast product concentration $\left(C_{\mathrm{I}}\right)$ observed at the input of the vessel is propagated to the output in time $\Delta t$. The situation is more complicated when a vessel junction is considered. The propagation depends on the flow direction (Fig. 1b and c). For the hepatic veins, the concentration at the bifurcation $C_{\mathrm{B}}$ is weighted by the corresponding blood flows. In any case, a recursive computation aimed at obtaining the concentration at each vessel, based on the earlier concentration in the preceding vessels, can be utilized. Data used at the inputs of HA or PV are concentration curves given as parameters of the model (they can be found e.g. in [12]). 


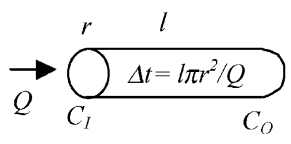

(a) $\quad C_{l}(t)=C_{O}(t+\Delta t)$

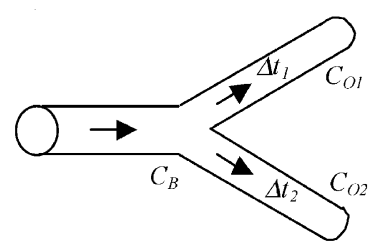

(b) $C_{B}(t)=C_{O 1}\left(t+\Delta t_{1}\right)=C_{O 2}\left(t+\Delta t_{2}\right)$

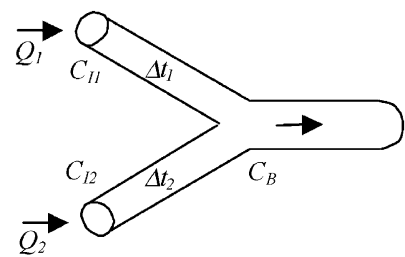

(c) $C_{B}(t)=\left(Q_{1} C_{11}\left(t-\Delta t_{1}\right)+Q_{2} C_{12}\left(t-\Delta t_{2}\right)\right) /\left(Q_{1}+Q_{2}\right)$

Fig. 1. Propagation of contrast material through the vessels, from main branches to arterioles and veinules. $C$ is the contrast material concentration, $Q$ - the blood flow, $l$ and $r$ - the length and the radius of the vessel, and $\Delta t$ - the time of transit of the blood (and contrast material) in the vessel.
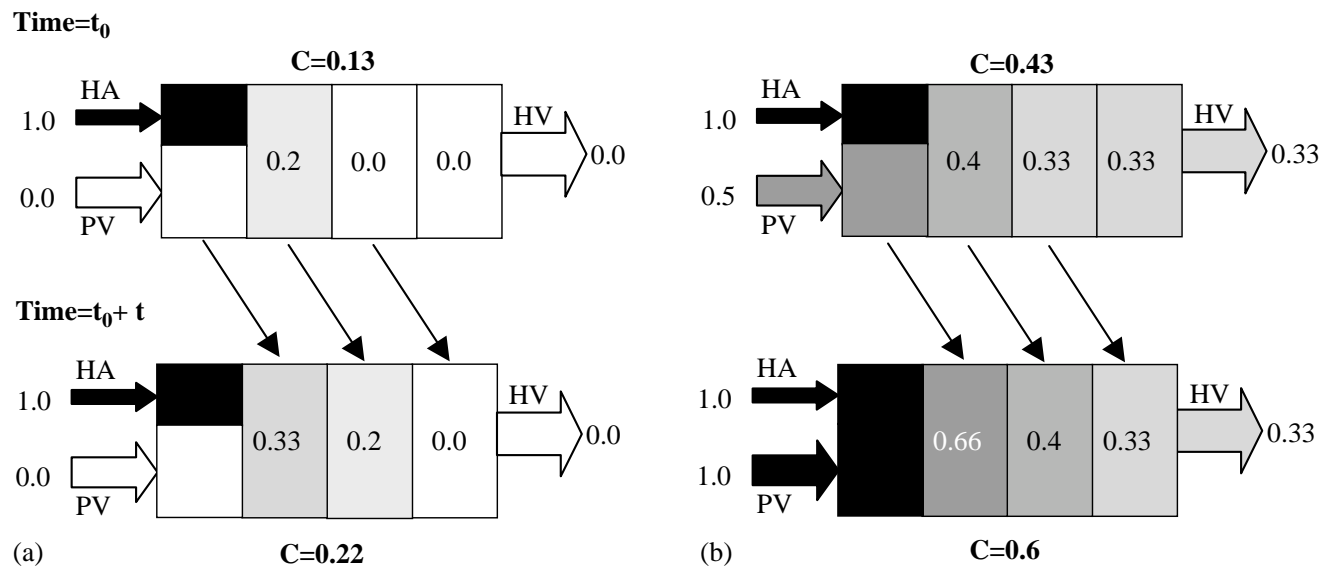

Fig. 2. Calculation of the contrast material concentration in a macro-cell at two time moments: (a) arterial phase, when contrast material arrives only through the hepatic artery - no contribution of portal vein (b) portal phase where the contribution of the portal vein is equivalent to the one of the artery. For example, on the (a) top scheme, the CM concentration is 1.0 (maximum possible value) in HA and 0.0 in PV. The resulting value of the first section's concentration is computed by weighting the respective supplies of HA (1/3 of the total flow) and PV (2/3). The mean concentration of the first section is then 0.33 at time $t_{0}$. The blood of the first section is then shifted to the next one at time $t_{0}+\Delta t$ (a-bottom). In this second scheme, the global concentration of CM in the macro-cell $(0.22)$ is the mean of the 4 sections $(0.33,0.33,0.2,0.0)$. Part (b) illustrates the similar process when CM arrives also in PV.

The propagation of blood and CM through capillaries is a complex process and a simplification has been performed. In a macro-cell, the volume of capillaries and extra-cellular space (which is defined as a fraction of the macro-cell volume) is divided into sections, whose size corresponds to the quantity of blood exchanged during the time interval imposed by the temporal resolution (Fig. 2). The concentration of the first section is calculated as weighted concentrations (according to the corresponding blood flows) of the two inputs. The organization of a macro-cell is based on a fixed-size queue (FIFO): each time the section's concentrations are shifted. The overall concentration of $\mathrm{CM}$ in the macro-cell is computed as an average concentration of sections. 


\subsubsection{Virtual $C T$}

The density inside each branch of the vascular network is therefore known at discrete times after the CM injection. Before generating CT slices, the density of all the voxels of the 3D liver volume has to be calculated and two cases can be encountered. First, the voxel is completely situated in a vessel, or in parenchyma. In that case, a mean density value is attributed to this voxel, and small fluctuations are added in order to simulate natural variations due to micro-vessels (capillaries). The second possibility for a voxel is to be located on the edge of a vessel: its density is then computed taking into account the respective volumes occupied by blood and parenchyma inside this voxel [2].

Based on the 3D density map representing the liver and its vascularization, the CT scan acquisition is carried out through the following steps: X-ray parallel projections are computed, using the Radon transform, projections are filtered in the Fourier domain, by a band-limited $|\omega|$ filter and a back-projection is applied to reconstruct the image.

\section{Results and discussion}

\subsection{Enhancement of a normal liver}

The parameters used to simulate the hepatic vascular network are given in Table 1. They correspond to standard values of physiological data and can be found in textbooks on hepatic anatomy and physiology. The final hepatic vascular network obtained after 50 growth cycles perfuse about 12,000 of macro-cells. The simulation of the HA, the PV and the HV took about $10 \mathrm{~h}$ on a PC (Pentium II $350 \mathrm{MHz}, 512 \mathrm{MB}$ RAM).

Fig. 3 (left) shows 3D simulated vascular trees in a healthy liver. The main branches of the HA and the PV are quite similar, due to the initialization phase where the few main branches of these two trees follow each others very closely, like in real hepatic vessels.

\subsection{Enhancement of a tumoral liver}

The 3D vascular model can be used to simulate hyper-vascular lesions like, for example, the Hepatocellular Carcinoma (HC), which represents the most common hyper-vascular hepatic malignant

Table 1

Main parameters of the model used in simulation of hepatic vascular network growth

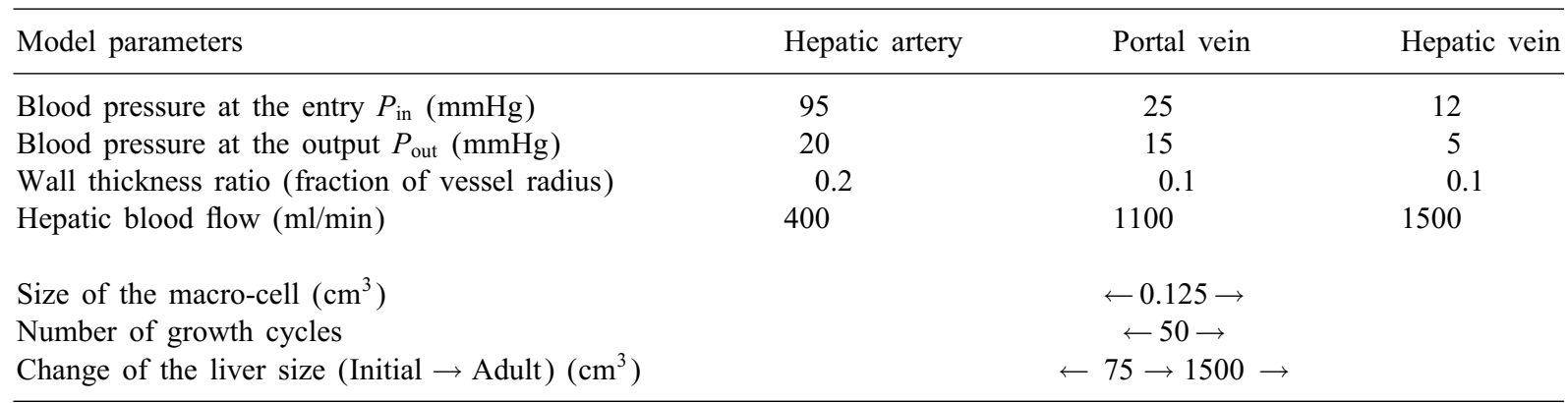




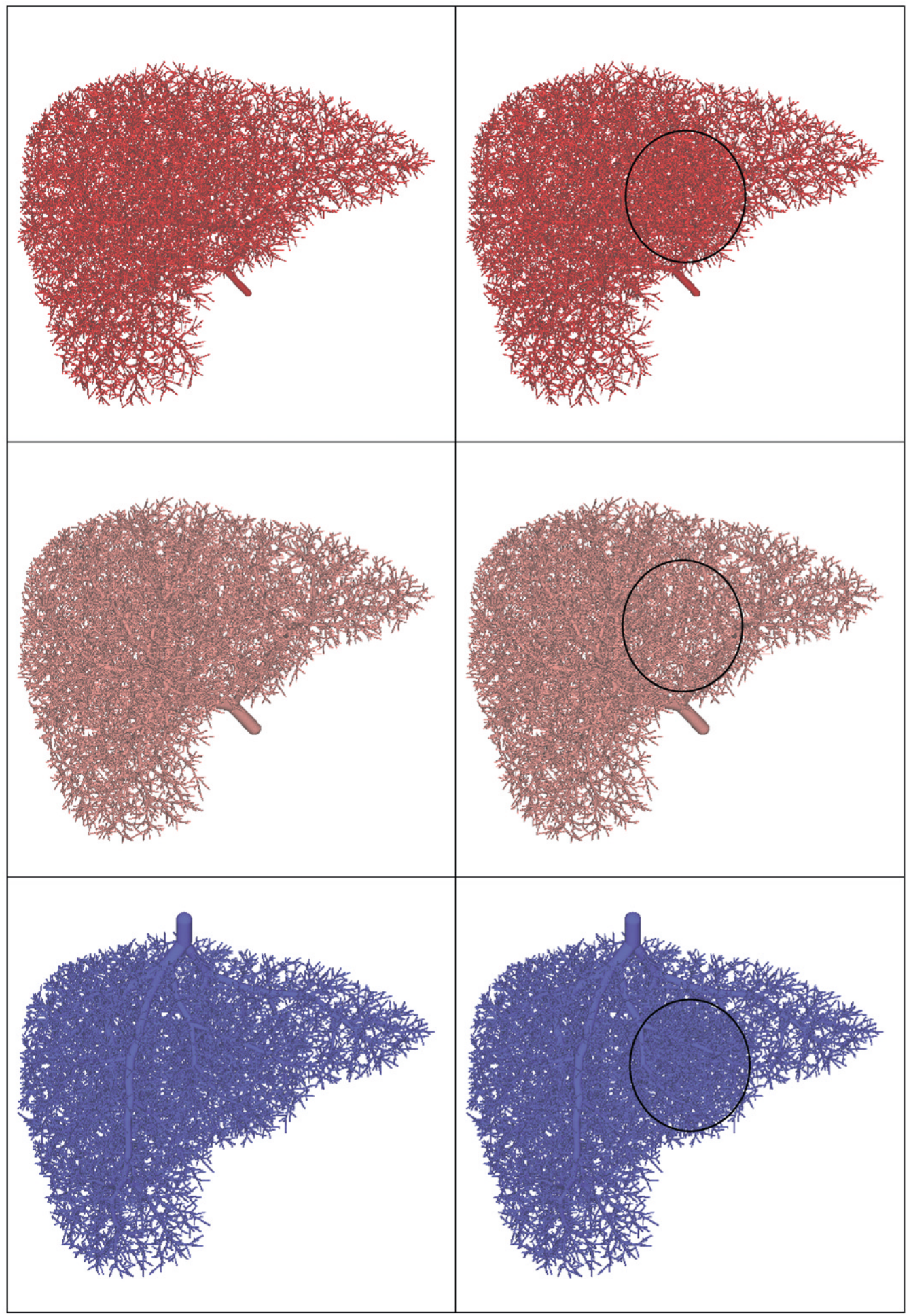

Fig. 3. Simulated hepatic vascular network: left column corresponds to healthy liver and tumoral one (hepatocarcinoma) is depicted in right column. Each tree is represented separately: Hepatic Artery (top), Portal Vein (center) and Hepatic Vein (bottom). Positions of a lesion are marked by circles. 


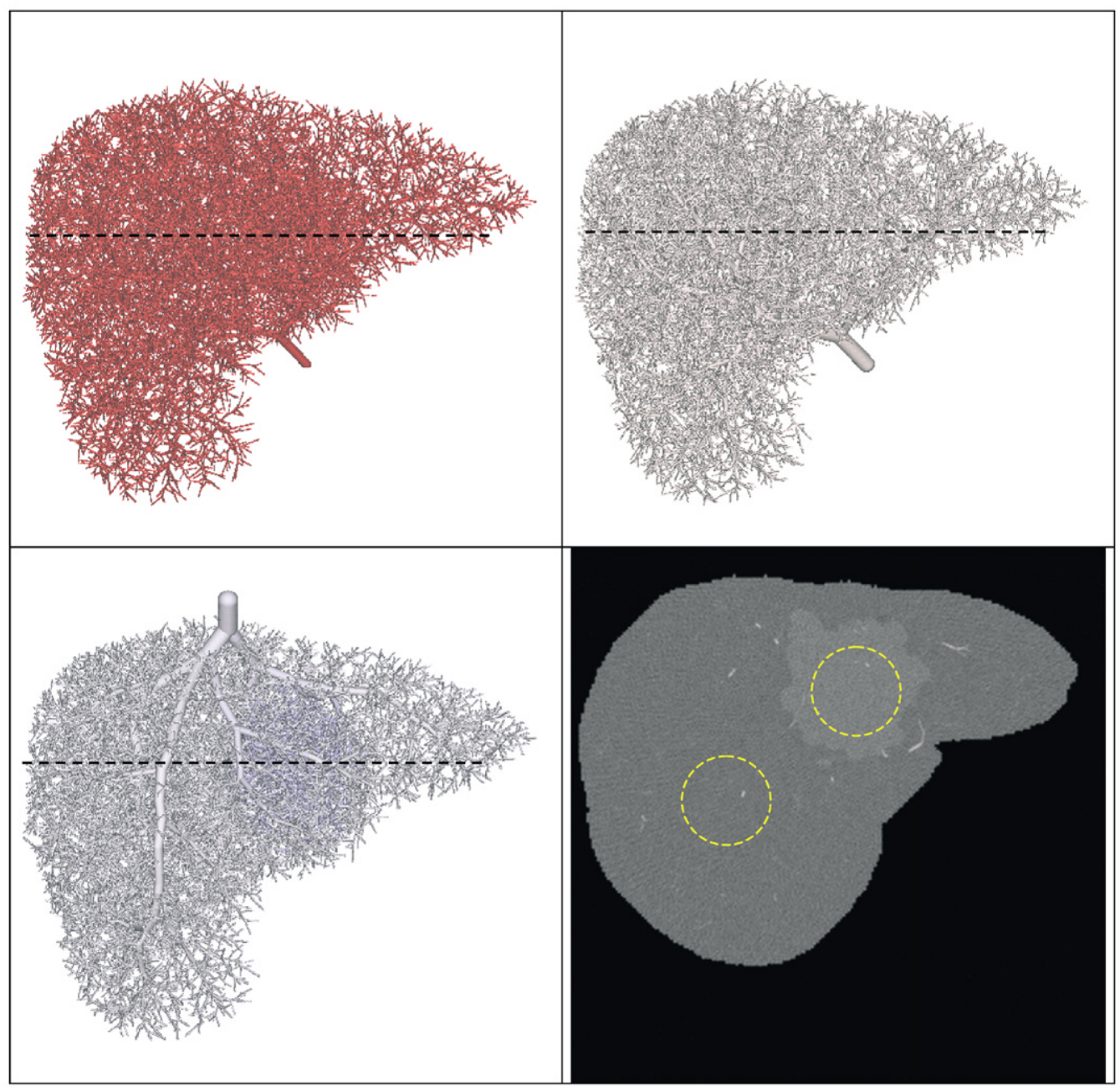

Fig. 4. Enhancement of a hyper-vascular lesion in 3D (HA, PV, HV) with the corresponding CT acquisition during the arterial phase. The scan position is represented by the horizontal line. Two circular ROI-s positions are also marked. The CT slice characteristics are: $512 \times 512$ matrix, 8-bit gray levels, $4 \mathrm{~mm}$ thickness.

tumor. The main differences between a normal vascularization and the HCs one is that this kind of tumor has only an arterial blood supply and does not receive portal venous blood flow, when normal hepatic tissue is perfused by the three hepatic trees (HA, PV, HV). To simulate a focal lesion in normal parenchyma, a second class of macro-cells is used. Few abnormal cells are introduced among normal ones, in a bounded area of the organ. Then, these tumoral macro-cells co-exist with the healthy ones, and evolve with them, in a succession of regeneration events (mitosis/necrosis). Some parameters associated to this second class of macro-cells are different of the normal ones: probability of mitosis, maximum local density and blood flow are increased in the pathological case. Moreover, when a new tumoral macro-cell appears, only a hepatic arteriole and a hepatic veinule sprout from the existing network, but no portal veinule. Fig. 3 (right) shows 3D simulated vascular 


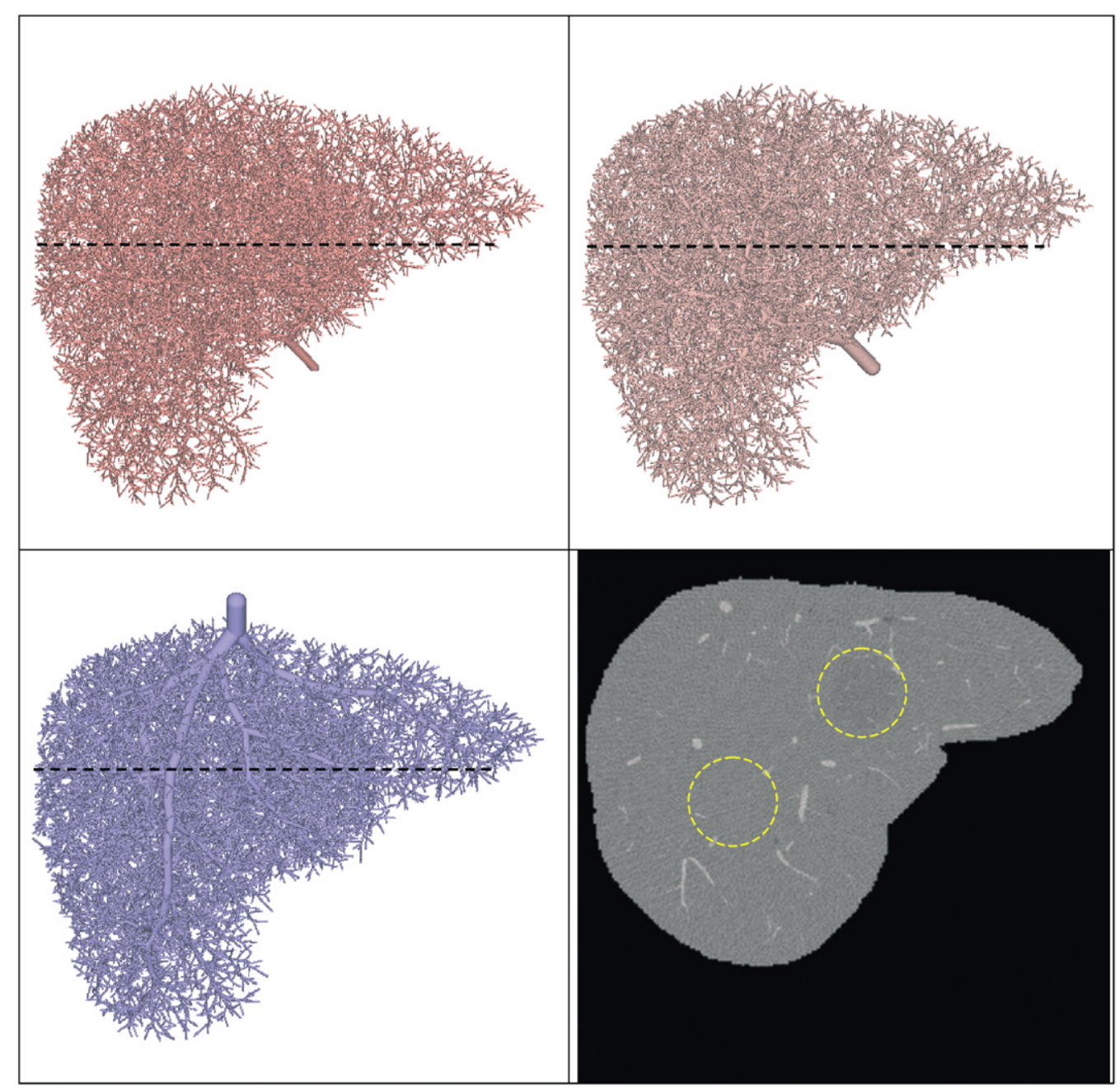

Fig. 5. The same results as in Fig. 4, during the portal phase.

trees in the tumoral liver. For both situations, HA, PV and HV are depicted separately. The localized hyper-vascularization is slightly visible. In Figs. 4 and 5, the arterial and portal phases of contrast product propagation are presented (enhanced 3D vascular trees and a corresponding CT slice). During the arterial phase, the lesion can be detected easily: it appears more intense than normal surrounding parenchyma. In Fig. 5, corresponding to the portal phase, the lesion becomes slightly hypo-dense when compared to normal tissue.

This enhancement is in agreement with what is observed during the classical radiological examination, realized for this kind of disease. During the first early acquisition phase (around 20-30 s after contrast infusion), $\mathrm{HC}$ receives highly concentrated $\mathrm{CM}$ coming from the HA, while the liver parenchyma is less enhanced because receiving less CM (PV does not contain CM yet), which leads to a high lesion conspicuity. Then, in a second phase, the normal parenchyma is strongly enhanced 


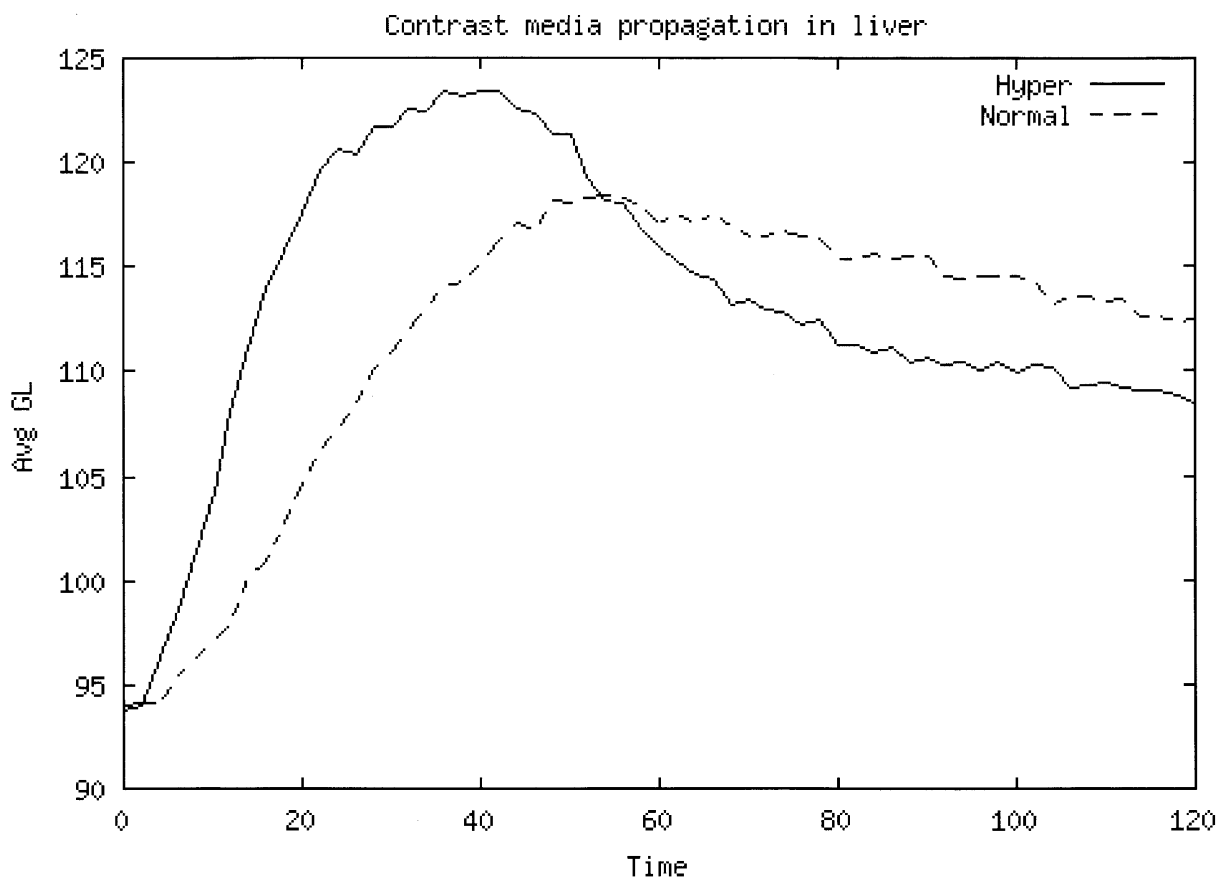

Fig. 6. Enhancement curves of the normal hepatic parenchyma and the hyper-vascular tumor after injection of a contrast material. The curves correspond to the mean value of enhancement calculated inside the ROIs represented in Figs. 4 and 5. Time is given in seconds.

due to the important flow of CM arriving through the PV (representing about $80 \%$ of the total flow) whereas the tumor CM supply (only arterial) diminishes. It leads to an inversion of the enhancement and to a decrease of conspicuity.

These results are confirmed on the enhancement curves showing the density evolution of the liver normal parenchyma and the hyper-vascular lesion (Fig. 6). The two regions of interest (ROIs) in which the density has been measured are represented in Figs. 4 and 5. The curves shows that the $\mathrm{CM}$ arrives first in the tumor, and later in the normal parenchyma, which is still explained by the fact that the tumor is perfused only by the HA, when the normal tissue is supplied by both the HA and the PV. After $40 \mathrm{~s}, \mathrm{CM}$ begins to decrease in the tumor, and then in the normal tissue. The portal phase is reached and the normal tissue density is slightly superior to the tumoral one, both of them being increased when compared to their respective levels before injection.

\section{Conclusion and perspectives}

In this paper, a model of dynamic CT liver enhancement is presented. It is composed of two main steps: modeling of the liver perfusion and modeling of dynamic CT. Compared to the original 
algorithm of 3D vascular modeling, the method has been improved in two important aspects: (1) the three hepatic trees (artery, portal vein and hepatic vein) are now modeled, allowing to simulate hyper and hypo-vascular lesions, and (2) the propagation of the contrast material in the microscopic vascularization is now possible, which allows to track the parenchymous enhancement, and not only the vessels one.

It has been showed that the combined models of liver perfusion and dynamic CT can reproduce several significant features of vascular pathological disturbances, and associated image modifications. Local vascular changes can easily be simulated, like for instance those related to the growth of a hyper-vascular tumor, which is generally supplied only by the artery and not by the portal vein. Series of time-stamped images can be synthesized, depending on the evolution of the contrast material in the vascular network and the parenchyma. Pathological vascular modifications can be followed over time in CT images. Results have been reported on the contrast evolution between the tumor and the normal hepatic parenchyma, measured in simulated images, which are in accordance with in vivo data, as well as enhancement curves.

This study emphasizes the great advantage of physiological vascular models compared to purely geometrical ones, which are not able to incorporate functional parameters. However, our vascular model can still be improved, especially at the microscopic level, where compartment models, based on patho-physiological knowledge, can be introduced. Future work will also deal with the evaluation of the influence of the injection protocols (changing the injection curves, and more precisely the injection time profile) on the images and on the typical time measurements (for instance, duration of the hepatic arterial phase). This model-based approach should be useful in the optimization of injection protocols and acquisition times, for in vivo examination.

\section{Summary}

Computer tomography (CT) imaging is the well-established tool used to detect and characterize lesions in the liver. Pathological vascular changes are often observed in such situations. The tumor conspicuity can be increased by the injection of contrast material $(\mathrm{CM})$, but it strongly depends on many physiological and acquisition parameters.

In order to better understand this complex interactions, a new computational approach, aimed at studying the hepatic enhancement in CT, is proposed. It relies on two models: (1) a vascular model based on physiological rules, is used to generate the $3 \mathrm{D}$ hepatic vascular network from the main branches (hepatic artery, portal vein and hepatic vein) to small arterioles and veinules; (2) the physical process of CT acquisition allows to synthesize timed-stamped series of images with varying parameters (e.g. spatial resolution), aimed at tracking the propagation of a contrast material through the vessel network and the parenchyma.

The hepatic tissue is composed of functional units, called macro-cells, which are evenly distributed in a 3D growing shape (obtained by reconstruction after segmentation of 2D in vivo images). The organ growth finishes when the external shape reaches the adult size. The characteristics of a macro-cell can depend on its class (tumoral or normal), time and position.

The vascular network, composed of three functionally connected trees (hepatic artery, portal vein and hepatic vein) perfuses the tissue: any appearing ischemic macro-cell is irrigated by a new vessel of each tree. The bifurcations are optimized to fulfill the minimal volume criteria. Trees are binary 
and made of a succession of branches and nodes. Blood flow coming from the two supplying vessels goes through the parenchyma and is collected by a hepatic veinule.

A contrast material, ideally mixed with the blood, propagates in the network, which results in changes of the vessels density. The fixed-size queue model is used to compute the contrast product concentration in each macro-cell.

Based on the 3D density representation, a filtered back-projection is used to synthesize timestamped CT images and enables to track the hepatic enhancement evolution.

The coupled models have been used to simulate the enhancement of a hyper-vascular tumor (e.g. Hepatocellular Carcinoma) at different acquisition times. These simulated images are in agreement with real acquisitions, showing a maximum conspicuity of the lesion during the arterial phase, with an hyper-density compared to normal parenchyma and a slightly inverted contrast, almost obscuring the lesion during the portal venous phase.

\section{References}

[1] J. Bézy-Wendling, A. Bruno, A 3-D dynamic model of vascular trees, J. Biol. Systems 7 (1) (1999) 11-31.

[2] J. Bézy-Wendling, M. Kretowski, Y. Rolland, W. Le Bidon, Toward a better understanding of texture in vascular CT scan simulated image, IEEE Trans. Biomed. Eng. 48 (1) (2001) 120-124.

[3] D. Bluemke, E. Fishman, Spiral CT of the liver, AJR 160 (1993) 787-792.

[4] R.L. Baron, Understanding and optimizing use of contrast material for CT of the liver, AJR 163 (1994) $323-331$.

[5] M.G. Frederick, B.L. McElaney, A. Singer, K.S. Park, E.K. Paulson, S.G. McGee, R.C. Nelson, Timing of parenchymal enhancement on dual-phase dynamic helical CT of the liver: how long does the hepatic arterial phase predominates?, AJR 166 (1996) 1305-1310.

[6] M.M. Walkey, Dynamic hepatic CT: how many years will it take' till we learn?, Radiology 181 (1991) $17-24$.

[7] V. Bonaldi, P. Bret, C. Reinhold, M. Atri, Helical CT of the liver: value of an early hepatic arterial phase, Radiology 197 (1995) 357-363.

[8] W.C. Small, R.C. Nelson, M.E. Bernardino, L.T. Brummer, Contrast-enhanced spiral CT of the liver: effect of different amounts and injection rates of contrast material on early contrast enhancement, AJR 163 (1994) 87-92.

[9] G.D. Dodd, R. Baron, Investigation of contrast enhancement in CT of the liver: the need for improved methods, AJR 160 (1993) 643-646.

[10] K.T. Bae, J.P. Heiken, J.A. Brink, Aortic and hepatic contrast medium enhancement at CT, prediction with a computer model, Radiology 207 (1998) 647-655.

[11] R. Materne, B.E. Van Beers, A.M. Smith, I. Leconte, J. Jamart, J.P. Dehoux, A. Keyeux, Y. Hormans, Non-invasive quantification of liver perfusion with dynamic computed tomography and a dual input one-compartmental model, Clin. Sci. 99 (2000) 517-525.

[12] S. Kim, J.H. Kim, J.K. Han, K.H. Lee, B.G. Min, Prediction of optimal injection protocol for tumor detection in contrast-enhanced dynamic hepatic CT using simulation of lesion to liver contrast difference, Comput. Med. Imag. Graph. 24 (2000) 317-327.

[13] R. Karch, F. Neumann, M. Neumann, W. Schreiner, A three-dimensional model for arterial tree representation, generated by constrained constructive optimization, Comput. Biol. Med. 29 (1999) 19-38.

[14] M. Zamir, On fractal properties of arterial trees, J. Theoret. Biol. 197 (4) (1999) 517-526.

[15] A. Kamiya, T. Togawa, Optimal branching structure of the vascular trees, Bull. Math. Biophys. 34 (1972) $431-438$.

[16] D.L. Cohn, Optimal systems: I. The vascular system, Bull. Math. Biophys. 16 (1954) 59-74.

[17] O. Hudlicka, M.D. Brown, S. Egginton, Angiogenesis: basic concepts and methodology, in: A. Halliday, B.J. Hunt, L. Poston M. Schachter (Eds.), An Introduction to Vascular Biology, Cambridge University Press, Cambridge, 1998, pp. 3-19.

[18] W. Schreiner, F. Neumann, M. Neumann, A. End, S.M. Roedler, S.H. Aharinejad, The influence of optimization target selection on the structure of arterial tree models generated by constrained constructive optimization, J. Gen. Physiol. 106 (1995) 583-599. 
[19] M.A. Changizi, C. Cherniak, Modeling the large-scale geometry of human coronary arteries, Can. J. Physiol. Pharmacol. 78 (2000) 603-611.

[20] M. Kretowski, Y. Rolland, J. Bézy-Wendling, J.L. Coatrieux, Fast algorithm for 3-D vascular tree modeling, Comput. Methods Programs Biomed. 2002, in print. 